\title{
Neural networks of aggression: ALE meta-analyses on trait and elicited aggression
}

Ting Yat Wong ${ }^{1,2}$, Azah Sid ${ }^{1}$, Tobias Wensing ${ }^{1,3}$, Simon B. Eickhoff ${ }^{4,5}$, Ute Habel ${ }^{1,6}$, Ruben C. Gur ${ }^{2}$, Thomas Nickl-Jockschat ${ }^{1,3,7,8}$

1. Department of Psychiatry, Psychotherapy and Psychosomatics, Faculty of Medicine, RWTH Aachen University, Germany

2. Brain Behavior Laboratory, Department of Psychiatry, Perelman School of Medicine, University of Pennsylvania, Philadelphia, PA, USA

3. JARA-BRAIN, Jülich-Aachen Research Alliance - Translation Brain Medicine, Aachen, Germany

4. Institute of Neuroscience and Medicine (INM-7), Research Center Jülich, Jülich, Germany

5. Institute of Systems Neuroscience, Medical Faculty, Heinrich Heine University Düsseldorf, Düsseldorf, Germany

6. JARA-Brain Institute I: Brain Structure Function Relationships, Aachen, Germany

7. Department of Psychiatry, Carver College of Medicine, University of lowa, lowa City, IA, USA

8. Iowa Neuroscience Institute, University of lowa, lowa City, IA, USA

* Address correspondence to Ting-Yat Wong, Department of Psychiatry, Psychotherapy and Psychosomatics, Faculty of Medicine, RWTH Aachen University, Pauwelsstraße 30,52074, Aachen, Germany; email: twong@ukaachen.de or tywong.one@outlook.com 


\begin{abstract}
There is considerable evidence that emotion dysregulation and self-control impairments lead to escalated aggression in populations with psychiatric disorders. However, convergent quantitative evidence on the neural network explaining how aggression arises is still lacking. To address this gap, peak activations extracted from extant functional magnetic resonance imaging (fMRI) studies were synthesized through coordinate-based meta-analyses. A systematic search in the PubMed database was conducted and $26 \mathrm{fMRI}$ studies met inclusion criteria. Three separate Activation Likelihood Estimation (ALE) meta-analyses were performed on i. Individual differences in trait aggression (TA) studies, ii. Individual differences in TA studies examining executive functioning, and iii. Elicited aggression (EA) studies across fMRI behavioral paradigms. Ensuing clusters from ALE meta-analyses were further treated as seeds for follow-up investigations on consensus connectivity networks (CCN) delineated from meta-analytic connectivity modeling (MACM) and resting-state functional connectivity (RSFC) to further characterize their physiological functions. Finally, we obtained a data-driven functional characterization of the ensuing clusters and their networks. This approach offers a boarder view of the ensuing clusters using a boarder network perspective. In TA, aberrant brain activations were found only in the right precuneus. Follow-up analyses revealed that the precuneus seed was within the
\end{abstract}


frontal-parietal network (FPN) associated with action inhibition, visuospatial processing and higher-level cognition. With further restricting to only experiments examining executive functioning, convergent evidence was found in the right rolandic operculum (RO), midcingulate cortex $(\mathrm{MCC})$, precentral gyrus ( $\operatorname{PrG})$ and precuneus. Follow-up analyses suggested that RO, MCC and PrG may belong to a common cognitive control network while the MCC seems to be the hub of this network. In EA, we only revealed a convergent region in the left postcentral gyrus. Follow-up CCN analyses and functional characterizations suggested that this region may also belong to the same cognitive control network found in the TA sub-analysis. Our results suggested that escalated aggression arises from abnormal precuneus activities within the FPN, disrupting the recruitment of other large-scale networks such as adaptive cognitive control network. Consequently, failure to recruit such a network results in an inability to generate adaptive responses, increasing the likelihood of acting aggressively. 


\section{INTRODUCTION}

Aggression is in the behavioral repertoire of virtually all animals and serves an evolutionarily adaptive purpose in survival, where it is hostile when defending threats and instrumental in competition for limited resources and increasing mating opportunities (Buss and Shackelford 1997). Aggression, however, can become maladaptive and destructive when it results in violating the social norms that maintain the order of a society. Several subgroups of psychiatric populations are often reported to be more susceptible to escalated aggression, including individuals with intermittent explosive disorder (Coccaro et al. 1998; Olvera 2002), schizophrenia (Milton et al. 2001; Douglas et al. 2009), psychopathy (Glenn and Raine 2008; Blair 2010), and antisocial or borderline personality disorders (Dolan 2010; Soloff et al. 2017).

Escalated aggression has been conceptualized as reflecting increased negative affect (i.e. anger) in the context of under-regulation of aggressive impulses (Davidson et al. 2000). The brain systems implicated in aggression were qualitatively postulated in a wider perturbed frontolimbic network functionally associated with affective processing, self-regulation, and reinforcement-based decision making (Davidson et al. 2000; Siever 2008; Coccaro et al. 2011; Blair 2016), comprising the amygdala, hypothalamus, periaqueductal gray, cingulate cortex, 
ventrolateral and medial prefrontal cortex, supplementary motor area, anterior insular cortex and striatum. Notably, other regions like posterior medial cortices are rarely discussed in the aggression literature but a recent large scale structural MRI study $(N=556)$ showed that the thickness of the precuneus and its contiguous regions were positively correlated with aggressive behavior in girls (Thijssen et al. 2015).

Instead of distinct networks for numerous cognitive functions, we noticed that this wider frontolimbic network is overlapping with a superordinate cognitive control network subserving various executive functions involving flexibility, working memory, response initiation and inhibition (Miller 2000; Niendam et al. 2012), suggesting that an executive functioning neural network may play an cardinal role in modulating aggressive behavior. Indeed, psychological studies have established a link between executive functions and aggression in healthy individuals (Hoaken et al. 2003; Denson et al. 2012). In accordance, the General Aggression Model (Anderson and Bushman 2002; Allen et al. 2018) proposed that aggressive acts are built on the (re-)appraisal processes while available cognitive resources play a key factor to bias the behavioral outcomes to either impulsive or thoughtful responses (Please note that the response can be either adaptive or maladaptive). Therefore, proper executive functioning signifies to ensure adaptive and non-aggressive behavior. More importantly, executive function impairment seems to be a core feature across 
psychopathology (Diamond 2013; Snyder et al. 2015). Recent neuroimaging meta-analyses have identified domain-nonspecific neural disruptions across psychiatric disorders in the salience (or anterior-cingulo-insular) network (Goodkind et al. 2015; McTeague et al. 2017). Evident for transdiagnostic impaired executive functioning leads to the question of whether there is a common disrupted cognitive control network in aggressive individuals with psychiatric diagnoses.

Previous functional magnetic resonance imaging (fMRI) studies of aggression focused primarily on cognitive processing including working memory (Kumari et al. 2006) and inhibition (Joyal et al. 2007), affective processes including perception of affective stimuli (Lee et al. 2009; Bobes et al. 2013) and emotional regulation (Kalnin et al. 2011; Schiffer et al. 2011) in aggressive individuals. These studies usually recruited participants with a history of violent incidents and heterogeneous psychiatric disorders who score high on measures of trait aggression (TA) by psychological scales. Other studies examined brain systems recruited when aggressive behavior is evoked. Such elicited aggression (EA) effects are studied through behavioral paradigms evoking aggression in a laboratory setting, for instances, the Taylor Aggression Paradigm (Taylor 1967), Point Subtraction Aggression Paradigm (Cherek et al. 1997) and violent video games (Mathiak and Weber 2006). These paradigms mimic aggressive responses of participants through provocation. These fMRI 
studies, however, use relatively small sample sizes, resulting in reduced statistical power and an increased likelihood of false positive results (Button et al. 2013). Furthermore, some studies abandon whole-brain inference spaces and adopt a region-of-interest approach to test a priori hypotheses. While this approach increases sensitivity for the chosen regions, it also entails the risk of limiting discovery.

Taken together, to identify a common disrupted neural network in trait aggressive individuals, we employed coordinate-based meta-analyses to quantitatively analyze the common aberrant brain activation pattern in aggressive individuals with psychiatric diagnoses. Specifically, we also investigate TA studies examining executive functioning (i.e., working memory and inhibition). To better understand the obtained clusters from trait aggressive individuals during the cognitive process, we also performed a meta-analysis on laboratory aggression paradigms. We hypothesized that both TA and EA studies should show convergent findings in the regions of the frontolimbic networks. Given that complex cognition and behavior, for example aggression, arise from dynamic interactions of distributed brain regions (Bressler and Menon 2010), a consensus connectivity network analysis was performed on each seed obtained from meta-analyses helps us estimating the coactivation pattern of a given seed region (i.e., how it connects with other brain regions) based on an independent healthy population and speculating dysfunctions in the aggressive 
population.

\section{METHOD}

\section{Literature Search and Data Acquisition}

A systematic literature search was conducted on the PubMed database (https://www.ncbi.nlm.nih.gov/pubmed/). We used the keywords "aggression", "aggressive", "violence", "violent", "intermittent explosive disorder" paired with "fMRI" or "functional magnetic resonance imaging" through June 2018. Further manuscripts were identified by reference tracing in the papers retrieved by the original search and textual reviews on the neurobiology of aggression (Hawkins and Trobst 2000; Anderson and Bushman 2002; Dolan 2010; Coccaro et al. 2011; Hoptman et al. 2011; Weiss 2012; Blair 2016). Studies were included if they 1) were peer-reviewed journal articles available in English, 2) reported whole-brain thresholded results; therefore, those results that restricted their analysis to a priori regions of interest (ROIs) were excluded, 3) reported coordinates in either the Montreal Neurological Institute (MNI) or Talairach standard stereotactic space, 4) for trait aggression (TA) studies, reported contrasts between aggressive psychiatric individuals with psychiatric disorders and their non-aggressive counterparts; psychiatric individuals refer to a 
formal diagnosis of either Axis I or II disorders including schizophrenia, intermittent explosive disorder and personality disorders, 5) for elicited aggression (EA) studies, reported either contrasts between induced aggressive and non-aggressive events on a virtual or real human targets or a correlation analysis of aggressive response and BOLD signal change within the experimental group, 6) recruited participants older than 18 . We were only interested in the general aberrant activation changes associated with aggression so hyper- and hypo-activations were pooled for the current meta-analyses. Figure 1 illustrates the steps of systematic search based on PRISMA criteria (Moher et al. 2015).

[Insert Figure 1 here]

In the context of ALE, the term "study" refers to a scientific publication while the term "experiment" refers to any single contrast analysis within a study (Laird et al. 2011). A total of 26 studies met our inclusion criteria (see Table 1 for details of the studies, Appendix I for full citations and Appendix II for all foci included in the current meta-analyses). Based on the original contrasts reported by the studies, we assigned them into two groups. Studies enrolling psychiatric individuals with a history of violence, e.g., violent schizophrenia patients compared to their non-aggressive counterparts, were categorized as studies on trait aggression ( $T A ; N=13$ ). Studies using a behavioral paradigm to compare BOLD responses 
during induced aggression were categorized as studies of elicited aggression (EA; $N=13$ ). $A$ follow-up analysis on $\mathrm{fMRI}$ tasks related to executive functioning was performed. A total of 569 participants with 67 experiments ( 23 executive functioning experiments) from TA studies and a total of 393 with 22 experiments from EA studies were included. All activation foci reported in a Talairach space were linearly transformed into a MNI space (Lancaster et al. 2007).

[Insert Table 1 here]

\section{Coordinate-Based Meta-Analysis: ALE}

The latest revised version of activation likelihood estimation (ALE) algorithm (Turkeltaub et al. 2002; Eickhoff et al. 2009, 2012) was implemented in MATLAB to perform our coordinate-based meta-analysis. Through modeling activation peak maxima were identified from the existing literature as 3-demensional probability distributions centered at the given coordinate rather than 1-dimensional points, the algorithm aims at quantitatively identifying topographic convergence across studies while accommodating spatial uncertainty. ALE is a well-established method adopted in multiple studies (Kohn et al. 2014; Nickl-Jockschat et al. 2015; Müller et al. 2017) and involves three main steps. First, to compensate for the spatial 
uncertainty due to a between-template and between-subject variance in most neuroimaging studies (Eickhoff et al. 2009), all the foci are modeled as centers of 3-demensional Gaussian probability distributions. Assuming a larger sample size is more likely to provide a reliable approximation of a "true" localization, studies are further weighted by the number of participants per study. Thus, a larger sample size leads to a smaller Gaussian distribution. Second, a modeled activation (MA) map is generated through combining the probabilities of all activation foci in a single experiment for each voxel (Turkeltaub et al. 2012). A voxel-wise ALE score is determined by calculating the union of these MA maps. Finally, to test significance, the yielded scores are compared with an empirical random spatial null-distribution among all MA maps yielding a $p$ value which was thresholded at $p<0.05$ with a cluster-level family-wise error (cFWE) correction (cluster-forming threshold at voxel-level: $p<0.001$ ) which provides the best compromise between sensitivty and specificity (Hopkins et al. 2016). Neuroanatomical labeling of the ensuing clusters was derived from the SPM anatomy toolbox (Eickhoff et al. 2005).

\section{Consensus Connectivity Networks (CCNs): Conjunction Analysis}

Unlike simple physiological process such as primary sensory perception, complex cognition and behavior, such as aggression, arise from dynamic interactions of distributed brain 
regions (Bressler and Menon 2010). Knowing the connectivity profile of a given brain region in healthy subjects helps to delineate the physiological functions of that region and allows to formulate data-driven hypotheses regarding dysfunctions, e.g., in population with escalated aggression. To identify the functional connectivity patterns that are consistently engaged across two different states of brain functions (i.e. task-dependent and task-independent), we performed a conjunction analysis across coactivation maps obtained from meta-analytic connectivity modeling (MACM) and resting-state functional connectivity (RSFC) using the strict minimum statistics with an individual seed (Amft et al. 2015). As the brain regions identified by this approach exhibit connectivity with the seed in two independent modalities of connectivity simultaneously, the ensuing networks can be regarded as especially robust (Nickl-Jockschat et al. 2015). The methodological details of MACM and RSFC are described later in this section. Surviving clusters from this conjunction analysis were, thus, functionally associated with both task-dependent and task-independent brain states. To reduce the likelihood of false positive findings, ensuing clusters smaller than 50 voxels were discarded. The CCN approach allows for inference on the involvement of clusters ensuing from our ALE meta-analyses in larger-scale cortical networks with reference to healthy populations. As stated above, our basic aim was the identification of neural networks in which the seed regions involve in to further characterize their physiological function. 
Task-Dependent Functional Connectivity: MACM. To characterize the functional connectivity among ensuing clusters from our original ALE meta-analyses, we determined the co-activation profile based on meta-analytic connectivity modeling (MACM). MACM was performed to examine the functional connectivity of a specific seed region (i.e. regions from our meta-analysis results) through investigating patterns of coactivation across large-scale neuroimaging experiments on the BrainMap Database (Fox et al. 2014). All experiments that activate at least one focus within the seed volume were retrieved. Subsequently, meta-analytic modeling using the ALE algorithm was employed to test for convergence across the foci reported in these experiments. With this approach, we aimed at a general neurobiological task-based co-activation profile for our ROIs. A cFWE-corrected threshold for multiple comparisons at $p<0.05$ (cluster-forming threshold at voxel-level: $p<0.001$ ) was adopted.

Task-Independent Functional Connectivity: RSFC. The same seeds obtained from our original ALE meta-analyses were also used for a resting-state functional connectivity (RSFC) analysis to assess a task independent modality of functional connectivity (Bzdok et al. 2015). Resting-state data were obtained from an open online database: the enhanced Nathan Kline Institute Rockland-sample (http://fcon 1000.projects.nitrc.org/indi/pro/nki.html). A total of 124 healthy subjects without a history of psychiatric or neurological disorders aged between 
20 and 75 years (mean age: $46.56 \pm 17.56$ years; 40 males, 84 females) was included. 404 echo-planar imaging (EPI) images of each subject were acquired on a Siemens TrioTim 3T scanner using blood-oxygen-level-dependent (BOLD) contrast [Multiband EPI sequence with acceleration factor 4 , repetition time $(T R)=1.4 \mathrm{~s}$, echo time $(T E)=30 \mathrm{~ms}$, flip angle $=65^{\circ}$, in-plane resolution $=2.0 \times 2.0 \mathrm{~mm}, 64$ axial slices with $2.0 \mathrm{~mm}$ thickness, covering the whole brain]. To allow for magnetic field saturation, the first four scans of each subject were discarded; the remaining 400 images were processed using SPM8 (http://www.fil.ion.ucl.ac.uk/spm/). First, images were corrected for head movements in a two-pass procedure, following by alignment to the initial volume and subsequently the mean of all volumes. Spatial normalization to a non-linear MNI152 template was applied to the mean image using the "unified segmentation" approach. The ensuing deformation was then applied to the individual EPI image. Finally, images were smoothed by a 5-mm FWHM Gaussian kernel and a band-pass filter between 0.01 and $0.08 \mathrm{~Hz}$ was applied to data. Time-courses of all voxels of a given seed of each individual subject and the time course of the entire seed were extracted as their first eigenvariate. Pearson correlation coefficients between the time series of the seeds and all other gray matter voxels across the whole brain were then computed to assess the RSFC. These correlation coefficients were transformed into Fisher's Z-scores and entered as dependent measures in a second level analysis of covariance, co-varying with age, gender and handedness. The RSFC results, consistent with 
MACM analysis, were thresholded at a cFWE corrected $p<0.05$ (cluster-forming threshold at voxel-level: $p<0.001)$.

\section{Functional Characterization through a Meta-Data Driven Approach}

Functional properties of the ensuing clusters and their CCNs were further assessed, using the "Behavioral Domain" and "Paradigm Class" meta-data categories assigned for each neuroimaging study stored in the BrainMap database (Laird et al. 2011). BrainMap taxonomy for "Behavioral Domain" codes mental processes into five major categories: action, cognition, emotion, interoception and perception; each category is further subdivided into sub-categories. "Paradigm Class" describes the categorization of the employed behavioral task (e.g. reward, Stroop or go/no-go, etc.) of a single study. Details about the taxonomy can be retrieved from the BrainMap website (http://www.brainmap.org/taxonomy/). In this study, we were interested in the precise functional associations of the ensuing clusters and their CCNs while a "Paradigm Class" may involve various "Behavioral domains"; thus, only functional categorization on "Behavioral Domains" was conducted. Both forward inferences and reverse inferences on the functional categorization using binomial test (FDR corrected $p$ $<0.05$ ) were performed (Eickhoff et al. 2011). The former, P(Activation|Domain), indicates the probability of activation in a neuroanatomical seed given a psychological process or 
behavioral task; the latter, $\mathrm{P}$ (Domain|Activation), tests the probability of observing a psychological process or behavioral task given a brain activation.

\section{RESULTS}

\section{Overall Analyses on Trait and Elicited Aggression}

Trait Aggression: Across 67 experiments in TA individuals, our ALE meta-analysis revealed a convergent cluster in the right precuneus (peak MNI: 4/-64/48; $k=116$ voxels; see Figure 2A). Functional characterization showed that the ensuing cluster was significantly associated with behavioral domains of action inhibition, reasoning, working memory, sexuality interoception, and motion vision perception. The functional coactivation pattern of the ensuing cluster was examined with MACM and RSFC. For more details see Table S1 and Figure S1 in the Supplementary Materials. The CCN of the precuneus seed (see Table 2A and Figure $3 \mathrm{~A}$ ) comprised the bilateral precuneus, bilateral middle occipital gyrus, bilateral middle frontal gyrus with the right clusters extending into the superior frontal gyrus. Functional characterization documented that the ensuing consensus network was associated with a wide range of the cognition domain including space, attention, explicit memory, 
working memory and reasoning plus other behavioral domains including interoception of sleep, action inhibition, visual perception of motion and shape (see Figure 4A for forward inference and reverse inference).

Elicited Aggression: Across 22 experiments in TA individuals, our ALE meta-analysis revealed a convergent cluster in the left postcentral gyrus (peak MNI: $-40 /-28 / 58 ; \mathrm{k}=99$ voxels; see Figure 2A). This result does not change after restricting only studies using the Taylor Aggression Paradigms. Functional characterization showed that the ensuing cluster was significantly and exclusively associated with action execution. The functional connectivity network of the ensuing cluster was examined with MACM and RSFC. For more details see Table S1 and Figure S1 in the Supplementary Materials. The CCN of the postcentral gyrus seed (see Table 2A for abbreviations and Figure 3A) comprised the bilateral postcentral gyrus extending into bilateral midcingulate cortex, bilateral precentral gyrus, right cerebellum, left insula, and left thalamus. Functional characterization documented that the ensuing consensus network was associated with pain perception as well as execution, preparation and imagination of actions (see Figure $4 \mathrm{~A}$ for forward inference and reverse inference).

\section{Subanalyses on Executive Functioning in Trait Aggression}


Activation Patterns during Executive Functioning: Across 23 experiments in TA studies during performing executive functions, our ALE meta-analysis revealed convergent clusters in the right precuneus (peak MNI: 5/-63/49; $k=171$ voxels), the left midcingulate cortex (peak MNI: -9/10/37; $k=110$ voxels), the rolandic operculum (peak MNI: 51/-9/14; $k=95$ voxels), the precentral gyrus (peak MNI: 42/4/50; $\mathrm{k}=91$ voxels). The functional connectivity network of the ensuing cluster was examined with MACM and RSFC. For more details see Table S1 and Figure S2 in the Supplementary Materials.

The $\mathrm{CCN}$ of the right rolandic operculum seed (see Table 2B for abbreviations and Figure 3B) comprised the right rolandic operculum and Heschls gyrus plus the bilateral insular cortices, superior temporal gyri, postcentral gyri, midcingulate cortices, and posterior-medial frontal gyri. Functional characterization documented that the ensuing consensus network was associated with interoception, action execution, motor learning, perception in pain and audition (see Figure 4B for forward inference and reverse inference).

The $\mathrm{CCN}$ of the right midcingulate cortex seed (see Table 2B for abbreviations and Figure 3B) comprised the bilateral midcingulate cortices, rolandic operculum, insula, thalamus, precentral gyri, inferior frontal gyri, and posterior-medial frontal gyri. The left hemisphere clusters also included the middle frontal gyrus, temporal pole and anterior cingulate cortex 
and the right hemisphere clusters also included the putamen, pallidum, postcentral gyrus and supramarginal gurus. Functional characterization documented that the ensuing consensus network was associated with action execution, language cognition, perception in pain and gustation (see Figure 4B for forward inference and reverse inference).

The CCN of the right precentral gyrus seed (see Table 2B for abbreviations and Figure 3B) comprised the bilateral precentral gyri, insula lobes, inferior frontal gyri, posterior-medial frontal gyri, and inferior parietal lobules. The right hemisphere clusters also included the middle frontal gyrus, superior temporal gyrus, supramarginal gyrus, middle occipital gyrus, middle temporal gyrus, midcingulate cortex and the right hemisphere clusters also included the superior parietal lobule. Functional characterization documented that the ensuing consensus network was associated with space and language cognition (see Figure 4B for forward inference and reverse inference).

Like the ALE seed from the overall result, the CCN of the precuneus seed in the sub-analysis (see Table 2B and Figure 3B) comprised the bilateral precuneus, bilateral middle occipital gyrus, bilateral middle frontal gyrus with the right clusters extending into the superior frontal gyrus. Functional characterization documented that the ensuing consensus network was associated with a wide range of the cognition domain including space, attention, explicit 
memory, working memory and reasoning plus other behavioral domains including interoception of sleep, action inhibition, visual perception of motion and shape (see Figure 4 for forward inference and reverse inference).

\section{DISCUSSION}

Extant fMRI literature in aggression can be broadly divided into two directions: (i) examining neural mechanisms in violent or aggressive samples with high trait aggression (TA) and (ii) examining neural mechanisms in elicited aggression (EA) through in a laboratory setting in a healthy sample. With the aid of data-driven coactivation analyses and functional characterization, the present coordinate-based meta-analysis quantitatively summarized the reported peak coordinates from these two directions to probe a generic functional neuroanatomy of aggression. We revealed that aggressive individuals, comparing to their non-aggressive counterparts, demonstrated aberrant activation changes in the right precuneus across different $\mathrm{fMRI}$ paradigms. With further restricting to experiments that required executive functions, our results displayed that disrupted activations associated with a cognitive control network in aggressive individuals comprised the right rolandic operculum (RO), midcingulate cortex (MCC), precentral gyrus ( $\mathrm{PrG}$ ) and precuneus. In provoked healthy 
subjects across EA experiments, the left postcentral gyrus was consistently involved.

\section{The Disrupted Precuneus-Related Networks in Trait Aggressive Individuals}

Surprisingly, aggressive individuals from TA studies were not characterized by significant abnormalities in limbic activation, but rather in the precuneus, which is part of the regulatory system and is implicated in self-consciousness and self-referential processes (Cavanna and Trimble 2006). Suppressing aggressive proneness initiated by external stimuli requires top-down cognitive control, with failure leading to escalated aggression (Davidson et al. 2000). Neuroanatomically, the precuneus is widely interconnected within the frontoparietal network (FPN) associated with elaborating highly-integrated and associative information (Cavanna and Trimble 2006). Functionally, our CCN analysis showed the precuneus seed was strongly coactivated with posterior parietal and lateral frontal cortices. The FPN has been shown to be involved in initiating and adjusting control according to feedback (Dosenbach et al. 2007, 2008; Zhang and Li 2012b). Recent evidence suggested the FPN may serve as a flexible hub facilitating adaptive task performance (Cole et al. 2013). Supporting the notion that abnormal precuneus activities are linked to aberrant activations in a wider part of the control network, our results demonstrated that the precuneus and its ensuing coactivation network were functionally associated with various cognitive domains 
related to executive functioning and higher-order cognition.

Arguably, the aberrant precuneus activations from our ALE analysis could reflect disruptions in default mode network (DMN) linking to aggression. For example, attenuated deactivation in the posterior medial cortex (PMC) of the DMN was documented in the high-psychopathy group during a task state (Freeman et al. 2015). The PMC, comprising the precuneus, posterior cingulate cortex and retrosplenial cortex, consume the highest level of glucose for brain energy metabolism during a resting state (Gusnard and Raichle 2001; Gur et al. 2009); when behavior becomes goal-directed, its consummation of glucose attenuates. Typically, the DMN comprises the bilateral temporal parietal junction, posterior cingulate cortex, middle temporal gyrus, precuneus and dorsomedial prefrontal cortex (dmPFC), while precuneus is considered as a functional core of the DMN (Utevsky et al. 2014). Notably, controversy on whether the precuneus is part of the DMN arose in the literature; few recent connectivity-based parcellation studies suggest that perhaps not the entire precuneus belong to the DMN (Zhang and Li 2012a; Bzdok et al. 2015).

\section{Cognitive Control Network in Aggression}

Diverse executive function domains may be implicated in a common superordinate 
fronto-cingulo-parietal network (Niendam et al. 2012). With a more detailed analysis of experiments examining executive functioning in TA studies, we further identified aberrant activations in the MCC, RO, and PrG as well as the precuneus. The follow-up CCN analyses showed that both the RO, PrG and MCC belonged to a common network while the MCC seemed to be the core hub of the network. Evidence showed that the MCC and its network not only integrates domains including negative affect, pain and cognitive control (Vogt 2005; Shackman et al. 2011), but also represents an important hub for regulating emotional reactivity (Kohn et al. 2014) as well as intentional motor control (Hoffstaedter et al. 2014). In accordance, our results showed that the MCC associated CCN was functionally associated with action execution and pain perception. Aggression is often prompted by underlying motives (Bushman and Anderson 2001). This MCC network leverages socioemotional information (i.e. pain, negative affect and cognitive control) for translating intentions into adaptive motor expressions (Paus 2001; Shackman et al. 2011; Shenhav et al. 2013). Thus, abnormal MCC activation in aggressive individuals may lead to failure of modulating inappropriate and aggressive reactions. Evaluating gains and losses could therefore promote the choice of a socially appropriate response guided by different motives (Shackman et al. 2011; Hillman 2013). A recent meta-analysis on youths with disruptive behavior, a population that often displays a wide range of phenotypical aspects of aggression such as hostile thoughts and violating social norms, showed that hypoactivation of the bilateral 
anterior MCC extending into SMA, rACC and medial prefrontal cortex with bilateral ventral caudate was found converging across studies (Alegria et al. 2016). Another study recruiting healthy youth revealed that the gray matter density of the anterior MCC was positively associated with hostile behavior (Nakagawa et al. 2017). Taking together, our findings provided evidence of a disrupted adaptive control network in aggressive psychiatric individuals, suggesting they are less likely to generate adaptive responses.

It is noteworthy that the derived CCN of the MCC was functionally associated with aspects of cognition related to language, suggesting that language might be engaged as a mechanism to mediate conflict and frustration in social situation thereby reducing risky elicited aggressive acts. Indeed there is evidence in children that language impairment contributes to aggression because of frustrating communication (Girard et al. 2014).

\section{Elicited Aggression in Healthy Samples: Issues of Paradigm Design}

To study neural mechanisms of aggression, paradigms including the Taylor aggression paradigm, point subtraction aggression paradigm and violent video games are adopted in a laboratory setting. Surprisingly, the right postcentral gyrus (PoG) was the only region identified in the meta-analysis across these aggression paradigms. In our CCN analyses, this 
region showed a similar coactivation pattern as the MCC, RO and $\operatorname{PrG}$ seeds, suggesting that it might be involved in a common cognitive control network, while the functional characterization showed that the CCN of the PoG was associated with action preparation. Critically, our results suggested that the laboratory aggression paradigm might not capture completely what the paradigms intend to study. For example, the Taylor aggression paradigm aims at provoking a negative affect (e.g. anger) in participants and instigate aggressive acts for others' punishment. It was expected that activation in limbic and prefrontal systems related to emotion and emotion regulation should take part during provocation. Such an inability is possibly caused by numerous inadequacies in these paradigms, such as external validity and experimental realism (Tedeschi and Quigley 1996). More recently, its methodological and analytic standardization were further criticized (Chester et al. 2018). These flexibilities eventually lead to inflation of false positive findings.

We would like to emphasize that none of the paradigms used in the elicited aggression studies elicited an actual act of violence. The need for standardized, often reductionist, paradigms that meet the technical requirements for functional imaging has often been discussed critically, especially when it comes to the study of complex behaviors, such as "aggression". This is a pertinent problem in neuroimaging. Of note, our approach to synthesize findings across distinct paradigms yields the great advantage to adjust for effects 
that are associated with a specific paradigm rather than the process or domain that is aimed to be studied. Despite this advantage, we would like to point out that these factors need to be considered in the interpretation of original studies as well as meta-analyses.

\section{The Common Neural Signature in Aggression}

Generally, aggression can be further categorized into either a reactive (also impulsive or hostile) and proactive (also premediated or instrumental) subtype (e.g., Dodge and Coie 1987; Barratt et al. 1999; Liu 2004). The former is described as an impulsive response driven by a strong emotional arousal under a perceived threat or provocation while the latter is described as a planned response driven by anticipation of reward. Real life aggression, however, tends to fall into a grey zone of this dichotomy. For example, a potential mass shooter plans for his/her revenge on the next day after he/she gets humiliated by peers. This example comprises elements of hostile emotions (anger out of humiliation) and deliberation which can be categorized as either subtypes of aggression. In fact, the dichotomy between reactive and proactive aggression was questioned by Bushman and Anderson (2001); they argued that a similar dichotomy, namely the automatic-controlled information processing dichotomy, can better describe the phenomenon with the emergence of modern cognitive theory. According to the General Aggression Model 
(Anderson and Bushman 2002; Allen et al. 2018), the (re)appraisal processes determine these subtypes of aggression. In other words, varying degrees of individual control can over their motivation and initiation of an action would result in largely different behavioral outcomes. The present meta-analysis aimed at examining the neural mechanism underlying a generic concept of aggression (i.e., above reactive nor proactive aggression) and indeed there was a common disrupted adaptive cognitive control network across aggressive individuals with psychiatric diagnoses. Our findings offer potentials to develop novel treatments to ameliorate aggressive symptoms in various psychiatric populations showing mixed forms of reactive and proactive aggression. Undeniably, aggression subtypes may involve domain specific neural networks, i.e. emotion related processing in reactive aggression and reward related processing in proactive aggression. However, our literature search did not yield a clear boundary that helps us to categorize studies into various subtypes of aggression. Future studies could focus on the domain-specific neural mechanisms in different subtypes of aggression.

\section{Methodological Limitations}

Our study has several limitations. First, while we tried our best to minimize heterogeneity across $\mathrm{fMRI}$ studies with strict inclusion criteria, the studies still varied on numerous 
variables including smoothing kernels, and analysis packages. These variables cannot be addressed separately due to methodological limitations; nevertheless ALE algorithm does estimate a spatial uncertainty per individual experiment to account for between-studies variability (Eickhoff et al. 2009). Second, included trait aggressive individuals (i.e. violent individuals with psychiatric diagnoses) varied on the demographical and medical background. However, we are looking for a least common denominator across heterogeneous individuals. Third, comparable to other meta-analyses our study is limited by the quality and scope of the available literature and the publication bias preferring positive results. Particularly, the small samples in each neuroimaging study might fail to randomize inter-subject variability such as personality traits (Simon et al. 2010).

\section{Conclusion}

The present coordinate-based meta-analysis identified not only the convergent brain regions associated with aggression but also their derived neural networks from a data-driven approach based on healthy participants. We argue that escalated aggression arises from abnormal precuneus activities within the FPN and/or DMN. This abnormality may further disrupt the recruitment of other large-scale networks such as adaptive cognitive control network comprising MCC, RO, PrG and PoG, resulting in inabilities to generate adaptive 
responses when needed. Generally, these results support the importance of the (re-)appraisal process in General Aggression Model while this abnormality may be linked to both reactive and proactive aggression. 


\section{End Note}

\section{Acknowledgements}

This study is supported by Deutsche Forschungsgemeinschaft (DFG, International Research Training Group IRTG2150). SBE is supported by the National Institute of Mental Health (R01-MH074457), the Helmholtz Portfolio Theme "Supercomputing and Modeling for the Human Brain" and the European Union's Horizon 2020 Research and Innovation Program under Grant Agreement Bi. 7202070 (HBP SGA1).

\section{Compliance with Ethical Standards}

The authors declare that they have no existing conflict of interest. 


\section{REFERENCES}

Alegria AA, Radua J, Rubia K (2016) Meta-analysis of fMRI studies of disruptive behavior disorders. Am J Psychiatry 173:1119-1130 . doi: 10.1176/appi.ajp.2016.15081089

Allen JJ, Anderson CA, Bushman BJ (2018) The General Aggression Model. Curr Opin Psychol 19:75-80 . doi: 10.1016/j.copsyc.2017.03.034

Amft M, Bzdok D, Laird AR, et al (2015) Definition and characterization of an extended social-affective default network. Brain Struct Funct 220:1031-1049 . doi: 10.1007/s00429-013-0698-0

Anderson CA, Bushman BJ (2002) Human aggression. Annu Rev Psychol 53:27-51 . doi: 10.1146/annurev.psych.53.100901.135231

Barratt ES, Stanford MS, Dowdy L, et al (1999) Impulsive and premeditated aggression: A factor analysis of self- reported acts. Psychiatry Res 86:163-173 . doi: 10.1016/S0165-1781(99)00024-4

Blair RJR (2010) Psychopathy, frustration, and reactive aggression: The role of ventromedial prefrontal cortex. Br J Psychol 101:383-399 . doi: 10.1348/000712609X418480

Blair RJR (2016) The neurobiology of impulsive aggression. J Child Adolesc Psychopharmacol 26:4-9 . doi: 10.1089/cap.2015.0088

Bobes MA, Ostrosky F, Diaz K, et al (2013) Linkage of functional and structural anomalies in the left amygdala of reactive-aggressive men. Soc Cogn Affect Neurosci 8:928-936 . doi: $10.1093 /$ scan/nss101

Bressler SL, Menon V (2010) Large-scale brain networks in cognition: emerging methods and principles. Trends Cogn Sci 14:277-290 . doi: 10.1016/j.tics.2010.04.004

Bushman BJ, Anderson CA (2001) Is it time to pull the plug on the hostile versus instrumental aggression dichotomy? Psychol Rev 108:273-279 . doi: 10.1037///0033-295X.108..1.273

Buss DM, Shackelford TK (1997) Human aggression in evolutionary psychological perspective. Clin Psychol Rev 17:605-619 . doi: 10.1016/S0272-7358(97)00037-8 
Button KS, Ioannidis JP a, Mokrysz C, et al (2013) Power failure: why small sample size undermines the reliability of neuroscience. Nat Rev Neurosci 14:365-376 . doi: $10.1038 / \mathrm{nrn} 3475$

Bzdok D, Heeger A, Langner R, et al (2015) Subspecialization in the human posterior medial cortex. Neuroimage 106:55-71 . doi: 10.1016/j.neuroimage.2014.11.009

Cavanna AE, Trimble MR (2006) The precuneus: A review of its functional anatomy and behavioural correlates. Brain 129:564-583 . doi: 10.1093/brain/awl004

Cherek DR, Moeller FG, Dougherty DM, Rhoades H (1997) Studies of violent and nonviolent male parolees: I. Laboratory and psychometric measurements of aggression. Biol Psychiatry 41:514-522 . doi: 10.1016/S0006-3223(96)00059-5

Chester DS, Lasko EN, Chester DS, House T (2018) Validating a standardized approach to the Taylor Aggression Paradigm. Soc Psychol Personal Sci. doi: $10.1177 / 1948550618775408$

Coccaro EF, Kavoussi RJ, Berman ME, Lish JD (1998) Intermittent explosive disorder-revised: Development, reliability, and validity of research criteria. Compr Psychiatry 39:368-376 . doi: 10.1016/S0010-440X(98)90050-5

Coccaro EF, Sripada CS, Yanowitch RN, Phan KL (2011) Corticolimbic function in impulsive aggressive behavior. Biol Psychiatry 69:1153-1159 . doi:

10.1016/j.biopsych.2011.02.032

Cole MW, Reynolds JR, Power JD, et al (2013) Multi-task connectivity reveals flexible hubs for adaptive task control. Nat Neurosci 16:1348-1355 . doi: 10.1038/nn.3470

Davidson RJ, Putnam KM, Larson CL (2000) Dysfunction in the neural circuitry of emotion regulation - A possible prelude to violence. Science (80- ) 289:591-594 . doi:

10.1126/science.289.5479.591

Denson TF, DeWall CN, Finkel EJ (2012) Self-control and aggression. Curr Dir Psychol Sci 21:20-25 . doi: 10.1177/0963721411429451

Diamond A (2013) Executive Functions. Annu Rev Psychol 64:135-168 . doi: 10.1146/annurev-psych-113011-143750 
Dodge KA, Coie JD (1987) Social-information-processing factors in reactive and proactive aggression in children’s peer groups. J Pers Soc Psychol 53:1146-1158 . doi: 10.1037/0022-3514.53.6.1146

Dolan MC (2010) What imaging tells us about violence in anti-social men. Crim Behav Ment Heal 20:199-214 . doi: 10.1002/cbm.771

Dosenbach NUF, Fair DA, Cohen AL, et al (2008) A dual-networks architecture of top-down control. Trends Cogn Sci 12:99-105 . doi: 10.1016/j.tics.2008.01.001

Dosenbach NUF, Fair DA, Miezin FM, et al (2007) Distinct brain networks for adaptive and stable task control in humans. Proc Natl Acad Sci 104:11073-11078 . doi: 10.1073/pnas.0704320104

Douglas KS, Guy LS, Hart SD (2009) Psychosis as a risk factor for violence to others: A meta-analysis. Psychol Bull 135:679-706 . doi: 10.1037/a0016311

Eickhoff SB, Bzdok D, Laird AR, et al (2012) Activation likelihood estimation meta-analysis revisited. Neuroimage 59:2349-2361 . doi: 10.1016/j.neuroimage.2011.09.017

Eickhoff SB, Bzdok D, Laird AR, et al (2011) Co-activation patterns distinguish cortical modules, their connectivity and functional differentiation. Neuroimage 57:938-949 . doi: 10.1016/j.neuroimage.2011.05.021

Eickhoff SB, Laird AR, Grefkes C, et al (2009) Coordinate-based activation likelihood estimation meta-analysis of neuroimaging data: A random-effects approach based on empirical estimates of spatial uncertainty. Hum Brain Mapp 30:2907-2926 . doi: 10.1002/hbm.20718

Eickhoff SB, Stephan KE, Mohlberg H, et al (2005) A new SPM toolbox for combining probabilistic cytoarchitectonic maps and functional imaging data. Neuroimage 25:13251335 . doi: 10.1016/j.neuroimage.2004.12.034

Fox PT, Lancaster JL, Laird AR, Eickhoff SB (2014) Meta-analysis in human neuroimaging: Computational modeling of large-scale databases. Annu Rev Neurosci 37:409-434 . doi: 10.1146/annurev-neuro-062012-170320

Freeman SM, Clewett D V., Bennett CM, et al (2015) The posteromedial region of the default mode network shows attenuated task-induced deactivation in psychopathic 
prisoners. Neuropsychology 29:493-500 . doi: 10.1037/neu0000118

Girard LC, Pingault JB, Falissard B, et al (2014) Physical aggression and language ability from 17 to 72 months: Cross-lagged effects in a population sample. PLoS One 9: . doi: 10.1371/journal.pone.0112185

Glenn AL, Raine A (2008) The neurobiology of psychopathy. Psychiatr Clin North Am 31:463-475 . doi: 10.1016/j.psc.2008.03.004

Goodkind M, Eickhoff SB, Oathes DJ, et al (2015) Identification of a common neurobiological substrate for mental Illness. JAMA Psychiatry 72:305-315 . doi: 10.1001/jamapsychiatry.2014.2206

Gur RC, Ragland JD, Reivich M, et al (2009) Regional differences in the coupling between resting cerebral blood flow and metabolism may indicate action preparedness as a default state. Cereb Cortex 19:375-382 . doi: 10.1093/cercor/bhn087

Gusnard DA, Raichle ME (2001) Searching for a baseline: Functional imaging and the resting human brain. Nat Rev Neurosci 2:685-694 . doi: 10.1038/35094500

Hawkins KA, Trobst KK (2000) Frontal lobe dysfunction and aggression: Conceptual issues and research findings. Aggress Violent Behav 5:147-157 . doi: 10.1016/S1359-1789(98)00033-0

Hillman KL (2013) Cost-benefit analysis: The first real rule of fight club? Front Neurosci 7:248 . doi: 10.3389/fnins.2013.00248

Hoaken PNS, Shaughnessy VK, Pihl RO (2003) Executive Cognitive Functioning and Aggression: Is It an Issue of Impulsivity? Aggress Behav 29:15-30 . doi:

10.1002/ab.10023

Hoffstaedter F, Grefkes C, Caspers S, et al (2014) The role of anterior midcingulate cortex in cognitive motor control: Evidence from functional connectivity analyses. Hum Brain Mapp 35:2741-2753 . doi: 10.1002/hbm.22363

Hopkins J, Cleveland WS, Mcgill R, et al (2016) Behavior, sensitivity, and power of activation likelihood estimation characterized bymassive empirical simulation. Neuroimage 137:70-85 . doi: 10.1038/d41586-017-07522-z 
Hoptman MJ, Antonius D, Kline N (2011) Neuroimaging correlates of aggression in schizophrenia: An update. Curr Opin Psychiatry 24:100-106 . doi: 10.1097/YCO.0b013e328342c8e0

Joyal CC, Putkonen A, Mancini-Marïe A, et al (2007) Violent persons with schizophrenia and comorbid disorders: A functional magnetic resonance imaging study. Schizophr Res 91:97-102 . doi: 10.1016/j.schres.2006.12.014

Kalnin AJ, Edwards CR, Wang Y, et al (2011) The interacting role of media violence exposure and aggressive-disruptive behavior in adolescent brain activation during an emotional Stroop task. Psychiatry Res - Neuroimaging 192:12-19 . doi: 10.1016/j.pscychresns.2010.11.005

Kohn N, Eickhoff SB, Scheller M, et al (2014) Neural network of cognitive emotion regulation - An ALE meta-analysis and MACM analysis. Neuroimage 87:345-355 . doi: 10.1016/j.neuroimage.2013.11.001

Kumari V, Aasen I, Taylor P, et al (2006) Neural dysfunction and violence in schizophrenia: An fMRI investigation. Schizophr Res 84:144-164 . doi: 10.1016/j.schres.2006.02.017

Laird AR, Eickhoff SB, Fox PM, et al (2011) The BrainMap strategy for standardization, sharing, and meta-analysis of neuroimaging data. BMC Res Notes 4:349 . doi: 10.1186/1756-0500-4-349

Lancaster JL, Tordesillas-Gutiérrez D, Martinez M, et al (2007) Bias between MNI and talairach coordinates analyzed using the ICBM-152 brain template. Hum Brain Mapp 28:1194-1205 . doi: 10.1002/hbm.20345

Lee TMC, Chan SC, Raine A (2009) Hyperresponsivity to threat stimuli in domestic violence offenders: A functional magnetic resonance imaging study. J Clin Psychiatry 70:36-45 . doi: 10.4088/JCP.08m04143

Liu J (2004) Concept analysis: Aggression. Issues Ment Health Nurs 25:693-714 . doi: $10.1080 / 01612840490486755$

Mathiak K, Weber R (2006) Toward brain correlates of natural behavior: fMRI during violent video games. Hum Brain Mapp 27:948-956 . doi: 10.1002/hbm.20234

McTeague LM, Huemer J, Carreon DM, et al (2017) Identification of common neural circuit 
disruptions in cognitive control across psychiatric disorders. Am J Psychiatry 174:676685 . doi: 10.1176/appi.ajp.2017.16040400

Miller EK (2000) The prefrontal cortex and cognitive control. Nat Rev Neurosci 1:59-65 . doi: $10.1038 / 35036228$

Milton J, Amin S, Singh SP, et al (2001) Aggressive incidents in first-episode psychosis. Br J Psychiatry 178:433-440 . doi: 10.1192/bjp.178.5.433

Moher D, Shamseer L, Clarke M, et al (2015) Preferred reporting items for systematicreview and meta-analysis protocols (PRISMA-P) 2015 statement. Syst Rev 4:1-9 . doi: 10.1186/2046-4053-4-1

Müller VI, Cieslik EC, Serbanescu I, et al (2017) Altered Brain Activity in Unipolar Depression Revisited. JAMA Psychiatry 74:47-55 . doi:

10.1001/jamapsychiatry.2016.2783

Nakagawa S, Takeuchi H, Taki Y, et al (2017) The anterior midcingulate cortex as a neural node underlying hostility in young adults. Brain Struct Funct 222:61-70 . doi: 10.1007/s00429-016-1200-6

Nickl-Jockschat T, Rottschy C, Thommes J, et al (2015) Neural networks related to dysfunctional face processing in autism spectrum disorder. Brain Struct Funct 220:2355-2371 . doi: 10.1007/s00429-014-0791-z

Niendam TA, Laird AR, Ray KL, et al (2012) Meta-analytic evidence for a superordinate cognitive control network subserving diverse executive functions. Cogn Affect Behav Neurosci 12:241-268 . doi: 10.3758/s13415-011-0083-5

Olvera RL (2002) Intermittent explosive disorder: Epidemiology, diagnosis and management. CNS Drugs 16:517-526 . doi: 10.2165/00023210-200216080-00002

Paus T (2001) Primate anterior cingulate cortex: Where motor control, drive and cognition interface. Nat Rev Neurosci 2:417-424 . doi: 10.1038/35077500

Schiffer B, Müller BW, Scherbaum N, et al (2011) Disentangling structural brain alterations associated with violent behavior from those associated with substance use disorders. Arch Gen Psychiatry 68:1039-1049 . doi: 10.1001/archgenpsychiatry.2011.61 
Shackman AJ, Salomons T V, Slagter HA, et al (2011) The integration of negative affect, pain and cognitive control in the cingulate cortex. Nat Rev Neurosci 12:154-167 . doi: $10.1038 /$ nrn2994

Shenhav A, Botvinick M, Cohen J (2013) The expected value of control: An integrative theory of anterior cingulate cortex function. Neuron 79:217-240

Siever LJ (2008) Neurobiology of aggression and violence. Am J Psychiatry 165:429-442 . doi: 10.1176/appi.ajp.2008.07111774

Simon JJ, Walther S, Fiebach CJ, et al (2010) Neural reward processing is modulated by approach- and avoidance-related personality traits. Neuroimage 49:1868-1874 . doi: 10.1016/j.neuroimage.2009.09.016

Snyder HR, Miyake A, Hankin BL (2015) Advancing understanding of executive function impairments and psychopathology: Bridging the gap between clinical and cognitive approaches. Front Psychol 6:328 . doi: 10.3389/fpsyg.2015.00328

Soloff PH, Abraham K, Burgess A, et al (2017) Impulsivity and aggression mediate regional brain responses in Borderline Personality Disorder: An fMRI study. Psychiatry Res Neuroimaging 260:76-85 . doi: 10.1016/j.pscychresns.2016.12.009

Taylor SP (1967) Aggressive behavior and physiological arousal as a function of provocation and the tendency to inhibit aggression. J Pers 35:297-310 . doi:

10.1111/j.1467-6494.1967.tb01430.x

Tedeschi JT, Quigley BM (1996) Limitations of laboratory paradigms for studying aggression. Aggress Violent Behav 1:163-177 . doi: 10.1016/1359-1789(95)00014-3

Thijssen S, Ringoot AP, Wildeboer A, et al (2015) Brain morphology of childhood aggressive behavior: A multi-informant study in school-age children. Cogn Affect Behav Neurosci 15:564-577 . doi: 10.3758/s13415-015-0344-9

Turkeltaub PE, Eden GF, Jones KM, Zeffiro TA (2002) Meta-analysis of the functional neuroanatomy of single-word reading: Method and validation. Neuroimage 16:765-780 . doi: 10.1006/nimg.2002.1131

Turkeltaub PE, Eickhoff SB, Laird AR, et al (2012) Minimizing within-experiment and within-group effects in activation likelihood estimation meta-analyses. Hum Brain Mapp 
33:1-13 . doi: 10.1002/hbm.21186

Utevsky A V, Smith D V, Huettel SA (2014) Precuneus is a functional core of the default-mode network. J Neurosci 34:932-940 . doi:

10.1523/JNEUROSCI.4227-13.2014

Vogt BA (2005) Pain and emotion interactions in subregions of the cingulate gyrus. Nat Rev Neurosci 6:533-544 . doi: 10.1038/nrn1704

Weiss EM (2012) Neuroimaging and neurocognitive correlates of aggression and violence in schizophrenia. Scientifica (Cairo) 2012:1-12 . doi: 10.6064/2012/158646

Zhang S, Li C shan R (2012a) Functional connectivity mapping of the human precuneus by resting state fMRI. Neuroimage 59:3548-3562 . doi: 10.1016/j.neuroimage.2011.11.023

Zhang S, Li CR (2012b) Functional networks for cognitive control in a stop signal task: Independent component analysis. Hum Brain Mapp 33:89-104 . doi:

10.1002/hbm.21197 
Figures

Figure 1. The PRISMA flowchart of the steps conducted in the meta-analyses

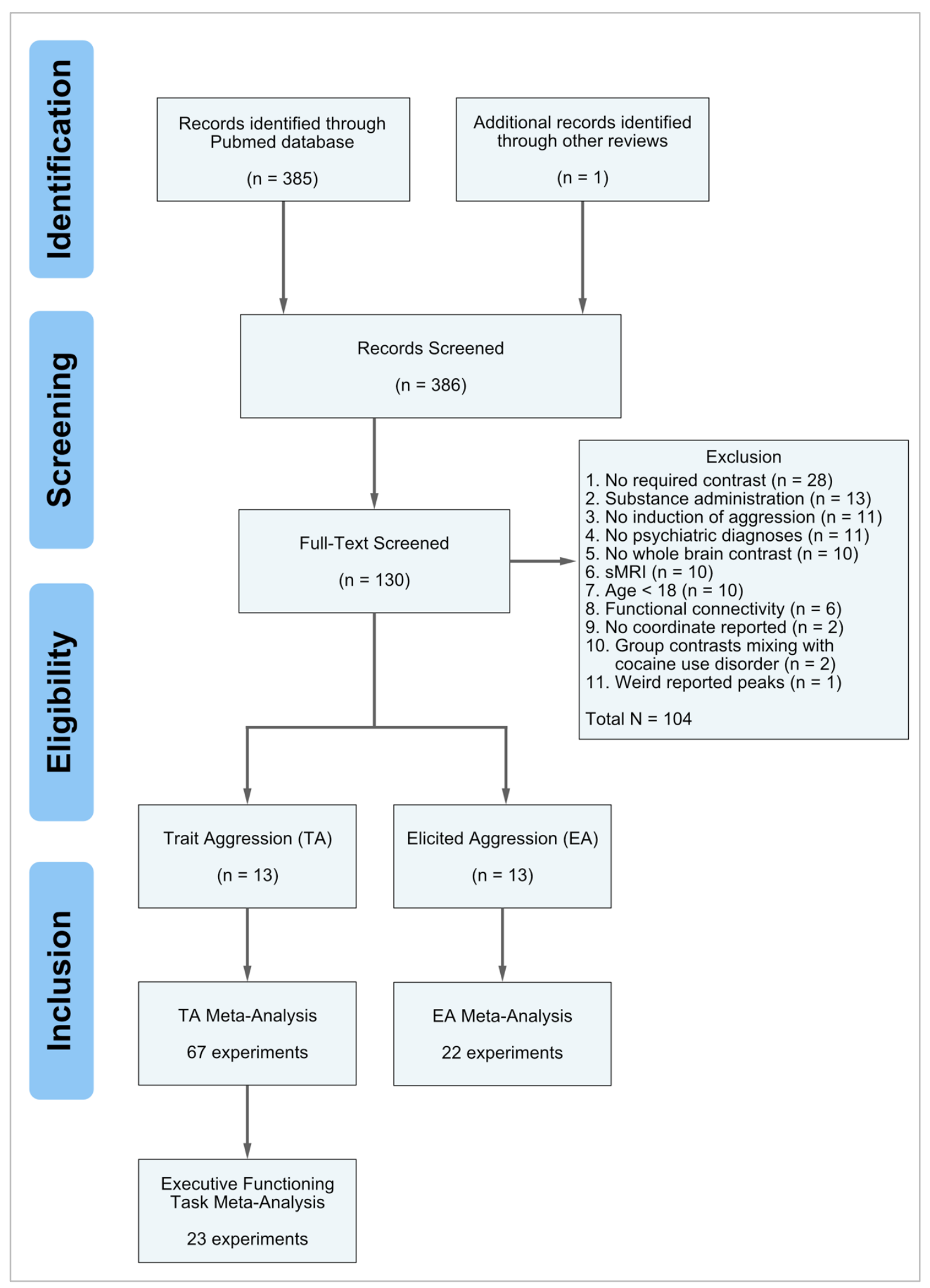


Figure 2. Convergent aberrant activation clusters from overall ALE analyses and subanalyses

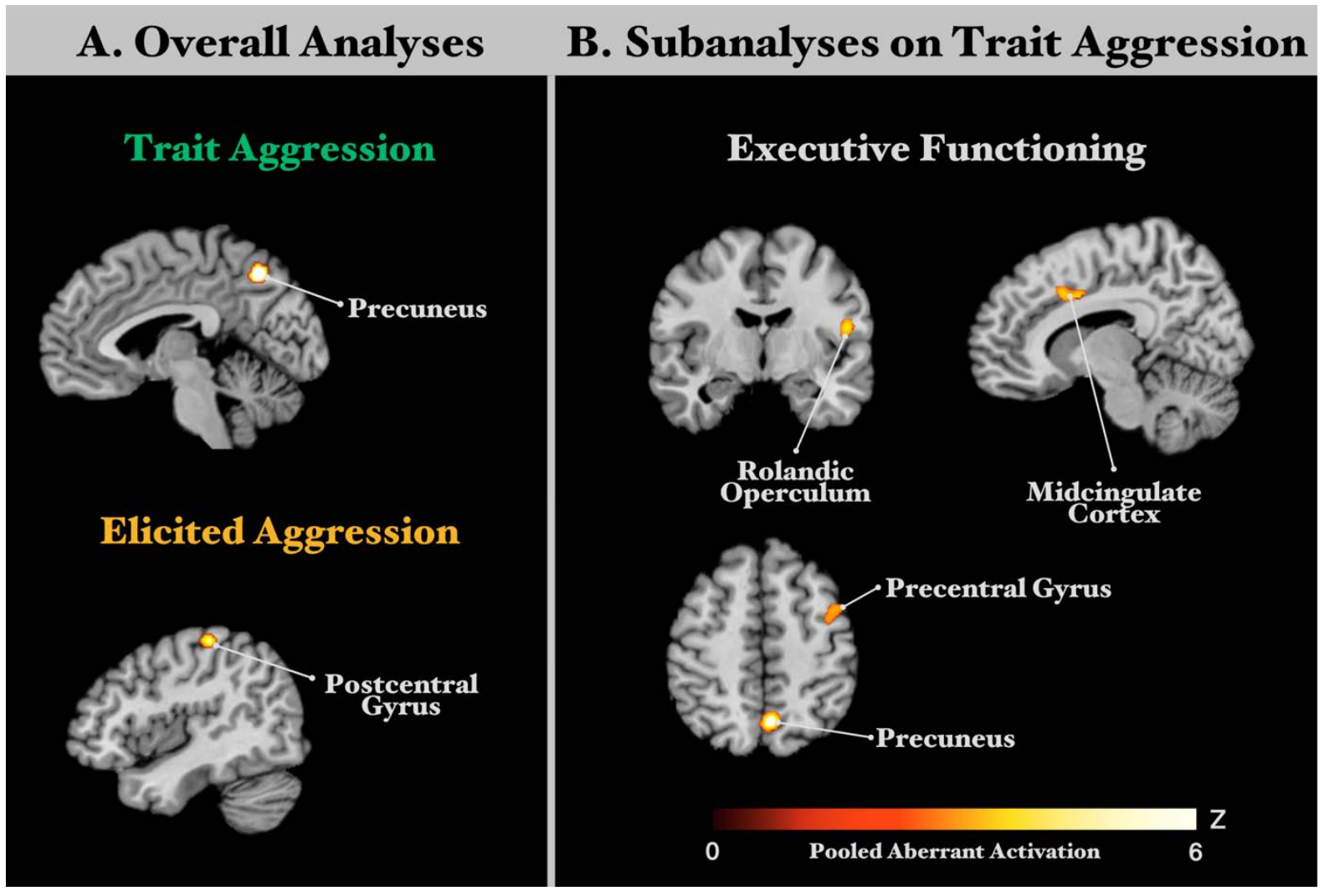

(A) Overall ALE analyses revealed significant clusters of convergent aberrant activation from all experiments in trait aggression (TA) studies and all experiments in elicited aggression (EA) studies. (B) Subanalyses focusing on executive functioning experiments in TA studies revealed significant clusters of convergent aberrant activation. All results survived a cluster-level FWE corrected threshold for multiple comparisons of $p<0.05$ (cluster-forming threshold at voxel-level, $p<0.001$ ). 
Figure 3. Consensus connectivity networks (CCNs) for each ensuing cluster from ALE analyses

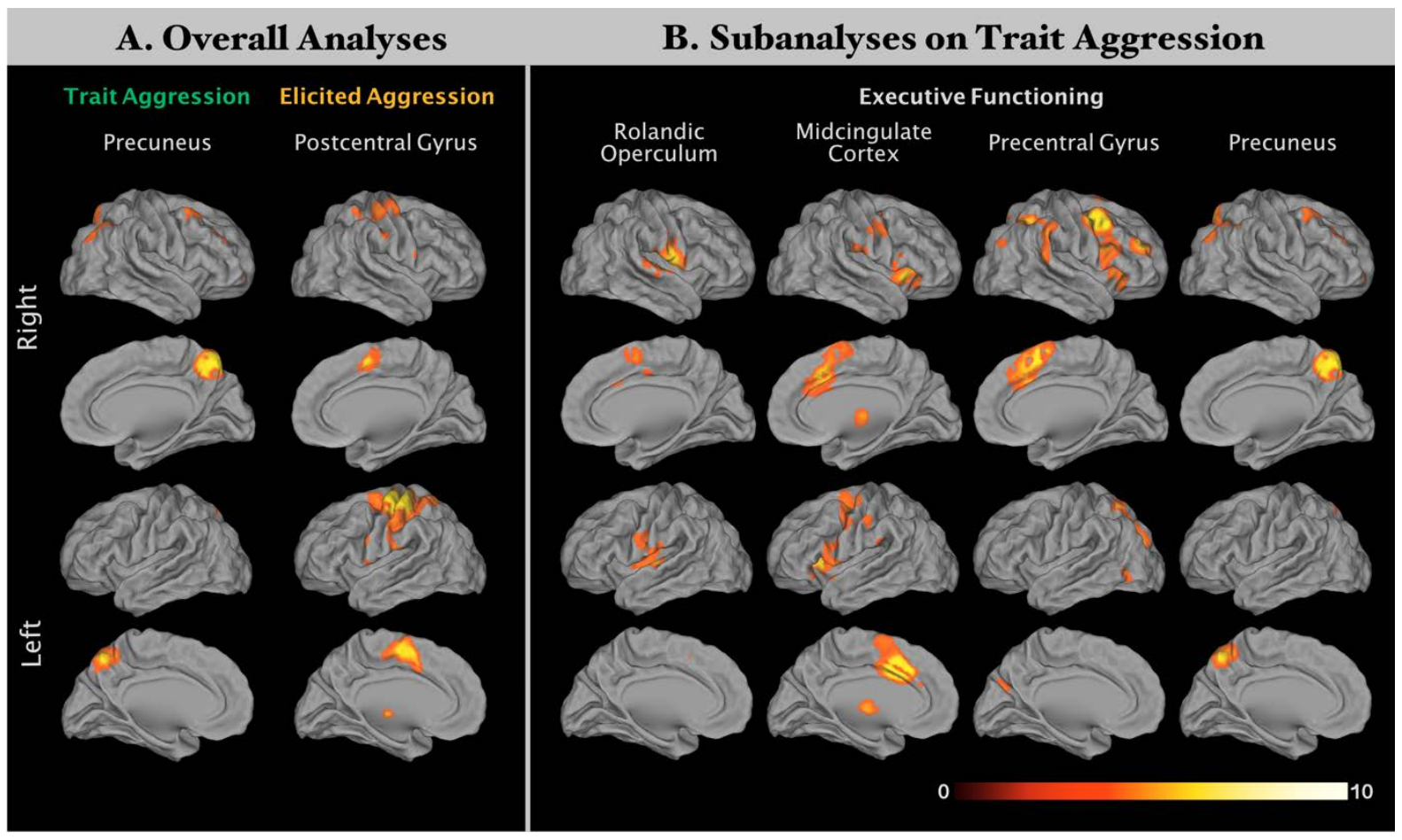

Overlapping functional connectivity patterns between meta-analytic connectivity modeling (MACM) and resting-state functional connectivity (RSFC) of each ensuing cluster from ALE analyses. Please refer to Table $\mathbf{2}$ for peak activations of each cluster. All results survived at a cluster-level FWE-corrected threshold for multiple comparisons of $p<0.05$ (cluster-forming threshold at voxel-level: $p<0.001$ ). The right bottom color bar represents $Z$-values. 
Figure 4. Forward inferences on behavioral domains in the consensus connectivity networks (CCNs)

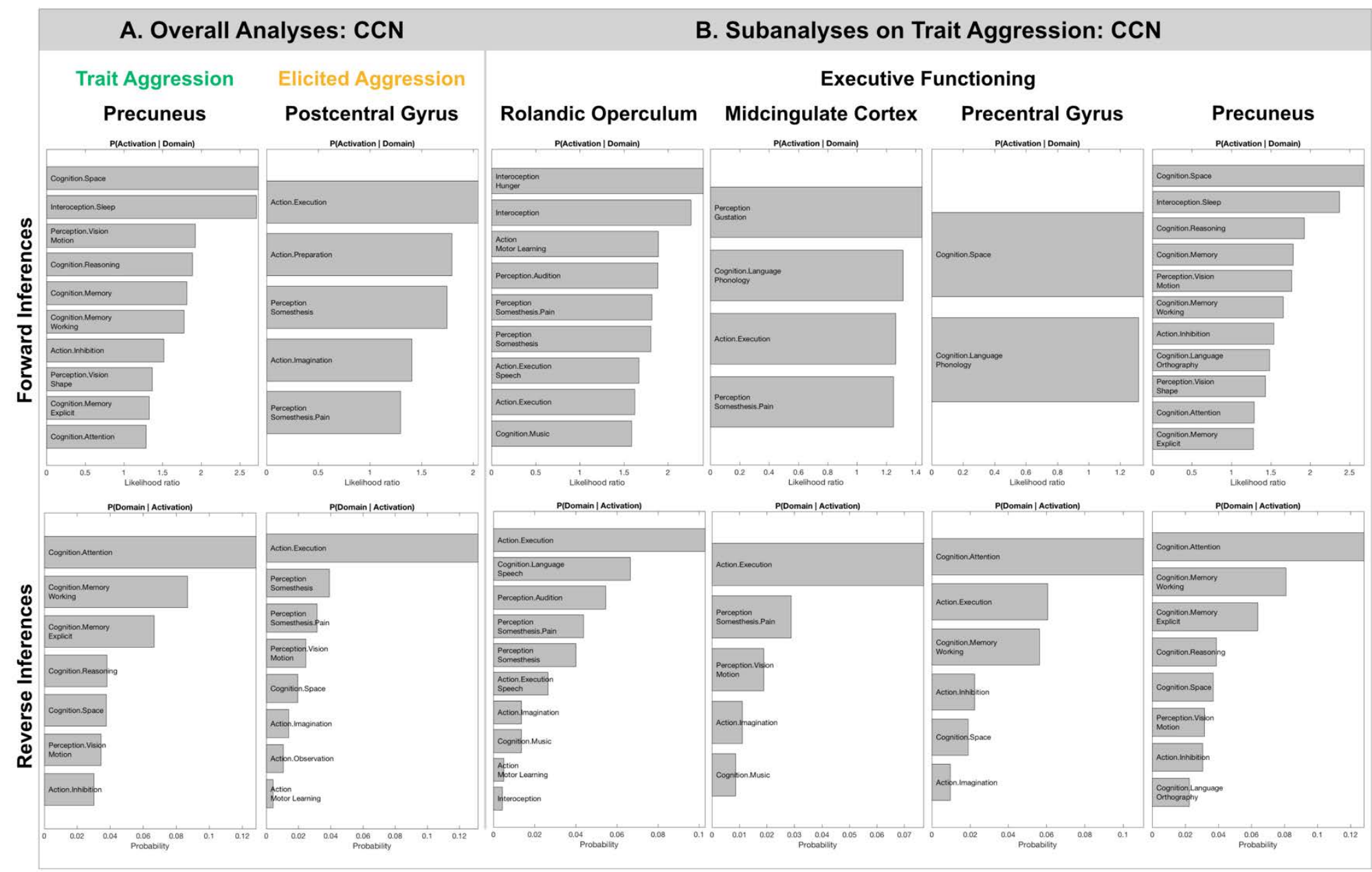

Forward inferences and reverse inferences on behavioral domains based on the BrainMap database. All results were significantly associated with the consensus connectivity networks (CCNs) with an FDR-corrected threshold for multiple comparisons of $p<0.05$. 
Tables

Table 1. Study samples and fMRI tasks. In an alphabetical order of first author's last name (See Appendix I for full references)

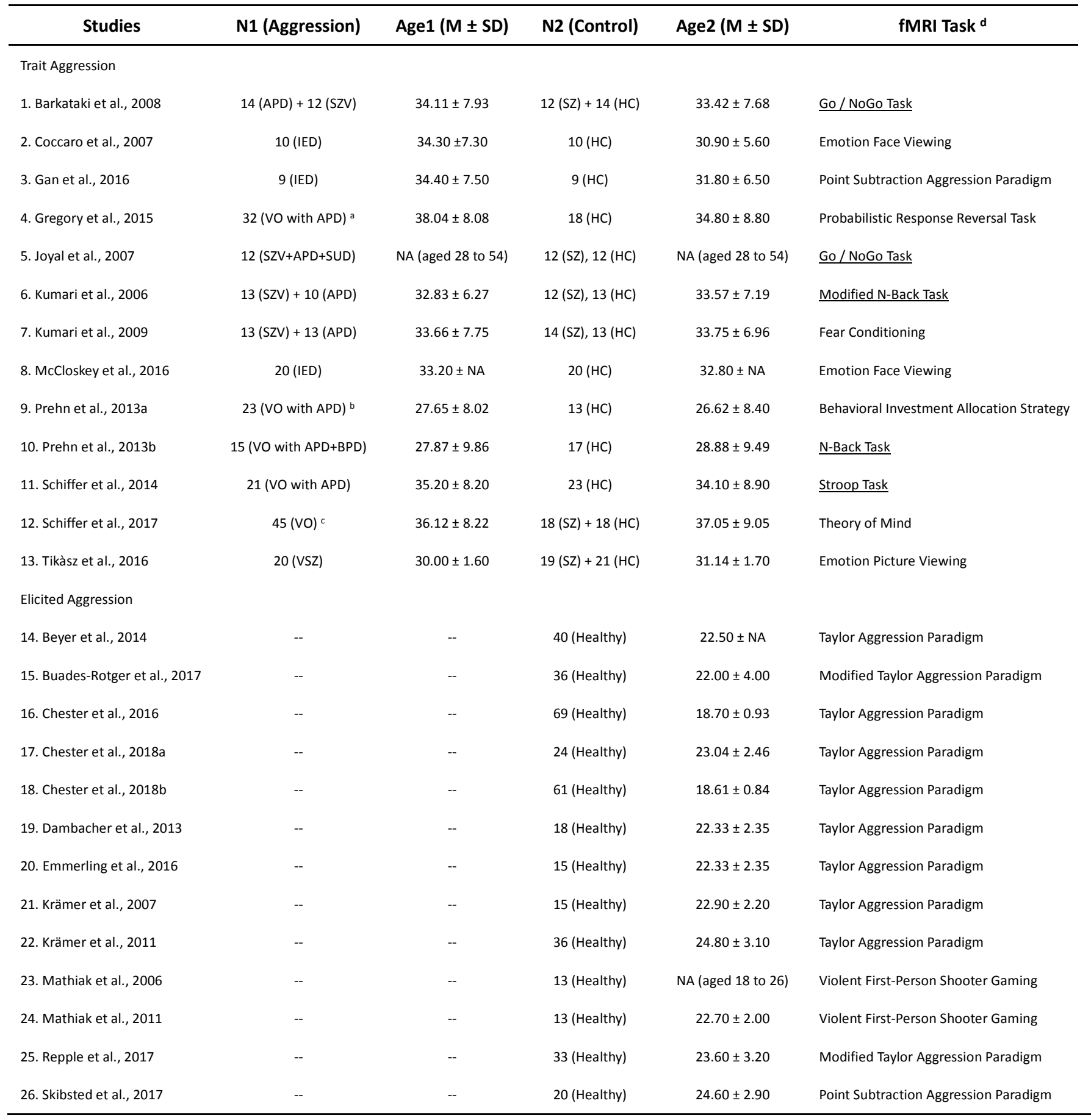

Notes:

a. 12 VO with APD and psychopathy and 18 VO with APD but not psychopathy

b. 23 VO with APD consists of 11 emotion hypo-reactivity and 13 emotion hyper-reactivity

c. 13 VO with SZ plus CD/APD, 16 VO with SZ and 16 VO with CD/APD

d. Underlined tasks were considered as executive functioning tasks

Abbreviations:

APD: Antisocial Personality Disorder with a Violent History, BPD: Borderline Personality Disorder, HC: Healthy Controls, IED: Intermittent Explosive Disorder, SZ: Schizophrenia 
without a Violent History, SZV: Schizophrenia with a Violent History, SUD: Substance Use Disorder, VO: Violent Offenders 
Table 2. Peak activations of the consensus connectivity networks (MACM $\cap$ RSFC)

$\begin{array}{lllllll}\text { Cluster } & \mathrm{k} \text { (voxels) Hemisphere } & \mathrm{x} & \boldsymbol{y} & \mathrm{z} & \text { Cluster Breakdowns }\end{array}$

\section{A. Overall Analyses}

\section{Trait Aggression: Precuneus Seed}

$\begin{array}{lllllll}1 & 788 & \text { R } & 9 & -63 & 50 & \text { R Precuneus; R Superior Parietal Lobule } \\ 2 & 596 & \text { L } & -7 & -65 & 51 & \text { L Precuneus; L Superior Parietal Lobule } \\ 3 & 281 & \text { R } & 37 & -64 & 42 & \text { R Angular Gyrus; R Middle Occipital Gyrus; R Inferior } \\ & & & & & & \text { Parietal Lobule; R Superior Parietal Lobule } \\ 4 & 231 & \text { R } & 28 & 10 & 53 & \text { R Middle Frontal Gyrus; R Superior Frontal Gyrus } \\ 5 & 99 & \text { R } & 34 & 33 & 39 & \text { R Middle Frontal Gyrus } \\ 6 & 87 & \text { R } & 28 & 58 & 3 & \text { R Middle Frontal Gyrus; R Superior Frontal Gyrus } \\ 7 & 57 & \text { L } & -25 & 9 & 53 & \text { L Middle Frontal Gyrus }\end{array}$

\section{Elicited Aggression: Postcentral Gyrus Seed}

\begin{tabular}{|c|c|c|c|c|c|c|}
\hline 1 & 3205 & $\mathrm{~L}$ & -38 & -26 & 53 & $\begin{array}{l}\text { L Postcentral Gyrus; L SupraMarginal Gyrus; L Superior } \\
\text { Parietal Lobule; L Inferior Parietal Lobule; L Superior } \\
\text { Temporal Gyrus }\end{array}$ \\
\hline 2 & 790 & $\mathrm{R}$ & 40 & -25 & 56 & $\begin{array}{l}\text { R Inferior Parietal Lobule; R Postcentral Gyrus; R Precentral } \\
\text { Gyrus; R SupraMarginal Gyrus; R Superior Frontal Gyrus }\end{array}$ \\
\hline 3 & 648 & $\mathrm{~L}$ & -7 & -8 & 54 & L Posterior-Medial Frontal; L Midcingulate Cortex \\
\hline 4 & 289 & $\mathrm{R}$ & 20 & -52 & -21 & R Cerebellum \\
\hline 5 & 181 & $\mathrm{~L}$ & -57 & 4 & 30 & L Precentral Gyrus \\
\hline 6 & 164 & $\mathrm{R}$ & 7 & -2 & 54 & R Posterior-Medial Frontal; R Midcingulate Cortex \\
\hline 7 & 81 & $\mathrm{~L}$ & -47 & -3 & 10 & L Insula Lobe; L Rolandic Operculum \\
\hline 8 & 64 & $\mathrm{R}$ & 58 & 8 & 24 & R Precentral Gyrus \\
\hline
\end{tabular}

\section{B Subanalyses on Executive Functioning in Trait Aggression}

1. Rolandic Operculum Seed

\begin{tabular}{|c|c|c|c|c|c|c|}
\hline 1 & 1325 & $\mathrm{R}$ & 53 & -11 & 16 & $\begin{array}{l}\text { R Rolandic Operculum; R Heschls Gyrus; R Superior } \\
\text { Temporal Gyrus; R Insula Lobe; R Postcentral Gyrus }\end{array}$ \\
\hline 2 & 1132 & $\mathrm{~L}$ & -49 & -13 & 15 & $\begin{array}{l}\text { L Superior Temporal Gyrus; L Insula Lobe; L Postcentral } \\
\text { Gyrus }\end{array}$ \\
\hline & 114 & $\mathrm{R}$ & 6 & -1 & 54 & R Posterior-Medial Frontal; R Midcingulate Cortex \\
\hline & 77 & $\mathrm{~L}$ & -4 & 7 & 49 & L Posterior-Medial Frontal; L Midcingulate Cortex \\
\hline
\end{tabular}

\section{Midcingulate Cortex Seed}

1

2159

$-40 \quad 5 \quad 23$

L Insula Lobe; L Inferior Frontal Gyrus; L Precentral Gyrus; L Middle Frontal Gyrus; L Rolandic Operculum; L Temporal Pole 


$\begin{array}{llllllll}2 & 962 & \text { L } & -7 & 7 & 45 & & \begin{array}{l}\text { L Midcingulate Cortex; L Posterior-Medial Frontal; L } \\ \text { Anterior Cingulate Cortex }\end{array} \\ 3 & 902 & \text { R } & 37 & 17 & 2 & \begin{array}{l}\text { R Insula Lobe; R Putamen; R Pallidum; R Rolandic } \\ \text { Operculum }\end{array} \\ 4 & 689 & \text { R } & 8 & 14 & 43 & \text { R Midcingulate Cortex; R Posterior-Medial Frontal } \\ 5 & 316 & \text { R } & 53 & -1 & 18 & \text { R Postcentral Gyrus; R Precentral Gyrus; R Inferior Frontal } \\ 6 & 216 & \text { L } & -49 & -46 & 23 & \text { L Rolandic Operculum } \\ 7 & 194 & \text { L } & -10 & -17 & 6 & \text { L Thalamus } \\ 8 & 193 & \text { R } & 10 & -16 & 6 & \text { R Thalamus } \\ 9 & 143 & \text { R } & 55 & -23 & 23 & \text { R SupraMarginal Gyrus }\end{array}$

\section{Precentral Gyrus Seed}

$13304-\mathrm{R}=41 \quad 16$

$\begin{array}{cccccc}2 & 1232 & \mathrm{~L} & -42 & 3 & 42 \\ 3 & 1165 & \mathrm{R} & 46 & -49 & 38\end{array}$

R Precentral Gyrus; R Insula Lobe; R Middle Frontal Gyrus;

R inferior Frontal Gyrus

L Precentral Gyrus; L inferior Frontal Gyrus

R Inferior Parietal Lobule; R Superior Temporal Gyrus; R

SupraMarginal Gyrus; R Middle Occipital Gyrus; R Middle Temporal Gyrus

$\begin{array}{ccccccl}4 & 830 & \mathrm{R} & 8 & 14 & 52 & \text { R Posterior-Medial Frontal; R Midcingulate Cortex } \\ 5 & 336 & \mathrm{~L} & -5 & 9 & 54 & \text { L Posterior-Medial Frontal } \\ 6 & 142 & \mathrm{~L} & -34 & -52 & 46 & \text { L Inferior Parietal Lobule; L Superior Parietal Lobule } \\ 7 & 121 & \mathrm{~L} & -36 & 20 & 4 & \text { L Insula Lobe }\end{array}$

\section{Precuneus Seed}

$\begin{array}{llllll}1 & 1276 & R & 18 & -63 & 48\end{array}$

$\begin{array}{ccccccl}2 & 639 & \text { L } & -8 & -64 & 52 & \text { L Precuneus; L Superior Parietal Lobule } \\ 3 & 264 & \text { R } & 28 & 9 & 53 & \text { R Middle Frontal Gyrus; R Superior Frontal Gyrus } \\ 4 & 127 & \text { R } & 36 & 33 & 39 & \text { R Middle Frontal Gyrus } \\ 5 & 113 & \text { L } & -27 & 9 & 55 & \text { L Middle Frontal Gyrus } \\ 6 & 100 & \text { R } & 29 & 58 & 4 & \text { R Middle Frontal Gyrus }\end{array}$

Clusters smaller than 50 voxels were excluded. Coordinates are reported in a Montreal Neuroimaging Institute (MNI) stereotaxic space. Macroanatomical locations are labeled by SPM Anatomy Toolbox (Eickhoff et al. 2005). 


\section{Appendix I: References - Studies included for ALE analysis}

\section{Studies on Trait Aggression (TA)}

1. Barkataki I, Kumari V, Das M, Sumich A, Taylor P, Sharma T (2008): Neural correlates of deficient response inhibition in mentally disordered violent individuals. Behav Sci Law. 26: 51-64.

2. Coccaro EF, McCloskey MS, Fitzgerald DA, Phan KL (2007): Amygdala and orbitofrontal reactivity to social threat in individuals with impulsive aggression. Biol Psychiatry. 62: 168178.

3. Gan G, Preston-Campbell RN, Moeller SJ, Steinberg JL, Lane SD, Maloney T, et al. (2016): Reward vs. retaliation-the role of the mesocorticolimbic salience network in human reactive aggression. Front Behav Neurosci. 10: 179.

4. Gregory S, Blair RJ, Ffytche D, Simmons A, Kumari V, Hodgins S, Blackwood N (2015): Punishment and psychopathy: $A$ case-control functional MRI investigation of reinforcement learning in violent antisocial personality disordered men. The Lancet Psychiatry. 2: 153-160.

5. Joyal CC, Putkonen A, Mancini-Marïe A, Hodgins S, Kononen M, Boulay L, et al. (2007): Violent persons with schizophrenia and comorbid disorders: A functional magnetic resonance imaging study. Schizophr Res. 91: 97-102.

6. Kumari V, Aasen I, Taylor P, Ffytche DH, Das M, Barkataki I, et al. (2006): Neural dysfunction and violence in schizophrenia: An fMRI investigation. Schizophr Res. 84: 144-164.

7. Kumari V, Das M, Taylor PJ, Barkataki I, Andrew C, Sumich A, et al. (2009): Neural and behavioural responses to threat in men with a history of serious violence and schizophrenia or antisocial personality disorder. Schizophr Res. 110: 47-58.

8. McCloskey MS, Phan KL, Angstadt M, Fettich KC, Keedy S, Coccaro EF (2016): Amygdala hyperactivation to angry faces in intermittent explosive disorder. J Psychiatr Res. 79: 3441.

9. Prehn K, Schlagenhauf F, Schulze L, Berger C, Vohs K, Fleischer M, et al. (2013a): Neural correlates of risk taking in violent criminal offenders characterized by emotional hypo- and hyper-reactivity. Soc Neurosci. 8: 136-147.

10. Prehn K, Schulze L, Rossmann S, Berger C, Vohs K, Fleischer M, et al. (2013b): Effects of emotional stimuli on working memory processes in male criminal offenders with borderline and antisocial personality disorder. World J Biol Psychiatry. 14: 71-78.

11. Schiffer B, Pawliczek C, Murüller B, Forsting M, Gizewski E, Leygraf N, Hodgins S (2014): Neural mechanisms underlying cognitive control of men with lifelong antisocial behavior. Psychiatry Res - Neuroimaging. 222: 43-51. 
12. Schiffer B, Pawliczek C, Müller BW, Wiltfang J, Brüne M, Forsting M, et al. (2017): Neural mechanisms underlying affective theory of mind in violent antisocial personality disorder and/or schizophrenia. Schizophr Bull. 43: 1229-1239.

13. Tikàsz A, Potvin S, Lungu O, Joyal CC, Hodgins S, Mendrek A, Dumais A (2016): Anterior cingulate hyperactivations during negative emotion processing among men with schizophrenia and a history of violent behavior. Neuropsychiatr Dis Treat. 12: 1397-1410.

\section{Studies on Elicited Aggression (EA)}

14. Beyer F, Münte TF, Krämer UM (2014): Increased neural reactivity to socio-emotional stimuli links social exclusion and aggression. Biol Psychol. 96: 102-110.

15. Buades-Rotger M, Beyer F, Krämer UM (2017): Avoidant Responses to Interpersonal Provocation Are Associated with Increased Amygdala and Decreased Mentalizing Network Activity. eNeuro. 4: e0337-16.2017.

16. Chester DS, DeWall CN (2016): The pleasure of revenge: Retaliatory aggression arises from a neural imbalance toward reward. Soc Cogn Affect Neurosci. 11: 1173-1182.

17. Chester DS, DeWall CN (2018a): Aggression is associated with greater subsequent alcohol consumption: A shared neural basis in the ventral striatum. Aggress Behav. 44: 285-293.

18. Chester DS, Dewall CN (2018b): Intimate partner violence perpetration corresponds to a dorsal-ventral gradient in medial PFC reactivity to interpersonal provocation. Soc Neurosci. . doi: 10.1080/17470919.2018.1430613.

19. Dambacher F, Sack AT, Lobbestael J, Arntz A, Brugman S, Schuhmann T (2013): Out of control: Evidence for anterior insula involvement in motor impulsivity and reactive aggression. Soc Cogn Affect Neurosci. 10: 508-516.

20. Emmerling F, Schuhmann T, Lobbestael J, Arntz A, Brugman S, Sack AT (2016): The role of the insular cortex in retaliation. PLoS One. 11: e0152000.

21. Krämer UM, Jansma H, Tempelmann C, Münte TF (2007): Tit-for-tat: The neural basis of reactive aggression. Neuroimage. 38: 203-211.

22. Krämer UM, Riba J, Richter S, Münte TF (2011): An fMRI Study on the Role of Serotonin in Reactive Agggression. PLoS One. 6: e27668.

23. Mathiak K, Weber R (2006): Toward brain correlates of natural behavior: fMRI during violent video games. Hum Brain Mapp. 27: 948-956.

24. Mathiak KA, Klasen M, Weber R, Ackermann H, Shergill SS, Mathiak K (2011): Reward system and temporal pole contributions to affective evaluation during a first person shooter video game. BMC Neurosci. 12: 66.

25. Repple J, Pawliczek CM, Voss B, Siegel S, Schneider F, Kohn N, Habel U (2017): From provocation to aggression: The neural network. BMC Neurosci. 18: 73. 
26. Skibsted AP, Cunha-Bang $S$ da, Carré JM, Hansen AE, Beliveau V, Knudsen GM, Fisher PM (2017): Aggression-related brain function assessed with the Point Subtraction Aggression Paradigm in fMRI. Aggress Behav. 43: 601-610. 